\title{
AN ABSTRACT VIRTUAL ENVIRONMENT TOOL TO ASSESS DECISION-MAKING IN IMPAIRED DRIVERS
}

\author{
Matthew Rizzo \\ Department of Neurology \\ University of Iowa \\ Iowa City, Iowa USA \\ E-mail: matthew-rizzo@uiowa.edu \\ Joan Severson, James Cremer, Kerri Price \\ Digital Artefacts, LLC \\ Iowa City, Iowa USA \\ E-mail: joan@digitalartefacts.com
}

\begin{abstract}
Summary: We describe design and pilot testing of software for evaluating decision-making abilities in drivers with neurological impairments. Instead of striving for visual realism, the virtual environment software is based on a more abstract representation that provides necessary visual cues in a singlescreen setting. Pilot tests were conducted on 16 subjects with neurological impairments (14 with focal brain lesions, two with Alzheimer's disease), and 16 neurologically normal subjects. Preliminary results are promising, suggesting that the PC-based virtual environment tool can distinguish decision-making impaired people where traditional neurological test batteries cannot.
\end{abstract}

\section{INTRODUCTION}

High-fidelity simulators offer promise as research tools to assess performance in at-risk drivers with cognitive impairments (Haraldsson et al., 1990; Brower et al., 1989; Madeley et al., 1990; Guerrier et al., 1995; Rizzo et al., 1997a; 2002). The standard design approach strives for computer generated photorealistic representations of real-world driving situations (Brooks, 1999), using multiple large display screens or head-mounted displays yielding 150- to 360degree fields of view, and providing optical flow and peripheral vision cues not easily achieved on a single small display. Yet the high cost and technical complexity of operating and maintaining these simulator systems, including software legacy, limits their use to large university or corporate research settings, and they cannot be practically deployed, e.g., in physician's offices, to assess drivers who are at risk for crashes due to cognitive impairments. Moreover, simulator scenario design has been ad hoc and unfocused.

Our experience with synthetic vision display system research for navigation and situational awareness supports a non-conventional design approach that uses (1) abstract (rather than photorealistic) representations of the virtual environment (Ellis, 1993; 2000), and (2) scenario design guided by cognitive neuroscience (to localize performance errors in specific cognitive domains that are crucial to the real-world task being simulated). We successfully implemented this approach in pilot studies aimed at testing critical Go/No-Go decisions by drivers with cognitive impairments caused by focal cerebral lesions. 


\section{METHOD}

To test Go/No-Go decision-making in cognitively impaired drivers we used a non-photorealistic representation of a 3D virtual space, with dynamic pictorial motion cues. Because our target display was a single computer monitor, the scenario and virtual environment had to be designed to present sufficient visual and pictorial cues to provide situational awareness in a 30- to 60degree field of view. The constrained field of view of small displays has been a design consideration for aviation display system researchers (Theunissen, 1997).

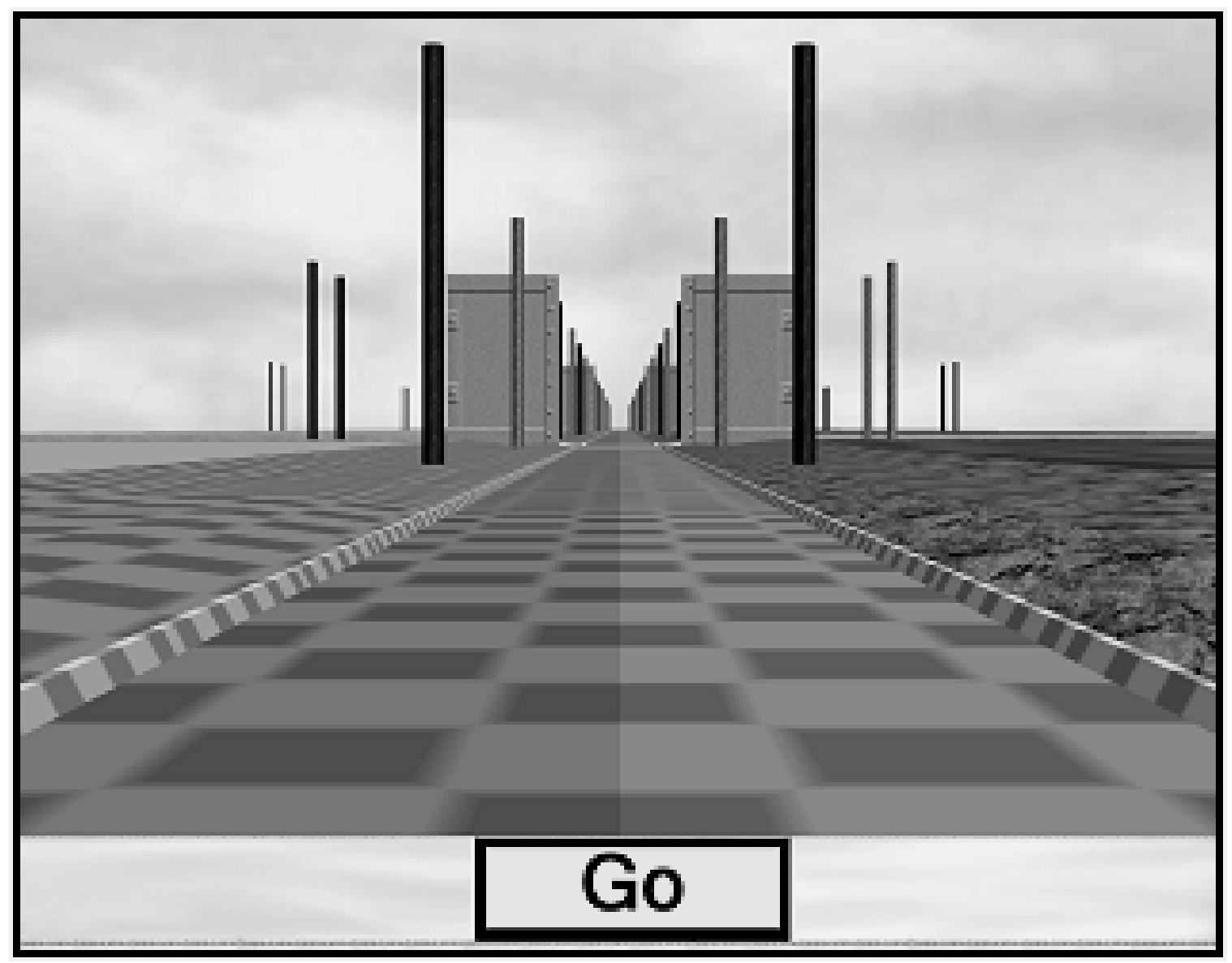

Figure 1. An image from the Go/No-Go tool

The virtual environment comprised a straight, flat, two-lane road intersected by an experimenterdesignated number of crossroads (see Figure 1). Driver inputs were recorded from a Microsoft SideWinder steering wheel and accelerator/brake hardware peripherals. All software for the experimental task was written in $\mathrm{C}++$. Graphics software relied on OpenGL and MultigenParadigm's Vega. The visual database was developed in Multigen-Paradigm's Creator. The pilot experiments were run on a Dell Optiplex GX240 computer with Nvidia GeForce3 graphics card and 21-inch CRT monitor. 


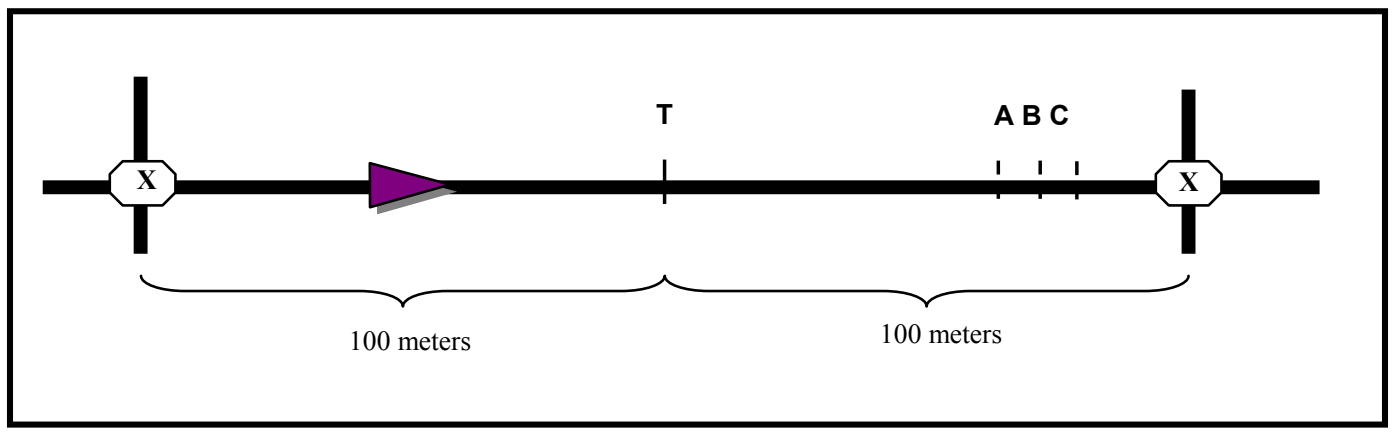

Figure 2. At point $T$, gate-closing trigger point A (easy), B (medium), or C (difficult) is computed, based on current speed, deceleration limit, and other parameters

In the Go/No-Go scenario, each subject drove through a series of intersections (marked by ' $\mathrm{X}$ 's in Figure 2) spaced 200 meters apart. Each intersection had gates that opened and closed. When the driver reached point T, 100 meters before an intersection, a green "Go" or red "Stop" signal appeared at the bottom of the display and a gate-closing trigger point (A, B, or C) was computed based on a deceleration constant, gate closure animation parameters, driver speed, and amount of time allotted to the driver to make a decision. Gates began closing the moment the subject reached the gate-closing trigger point. The allowed decision times (braking distances) were "easy," "medium," or "difficult" (shortest possible braking distance). Approximately 1/3 of the gates were represented at each difficulty level, and difficulty levels were randomized so as to be unpredictable to the driver.

Gates closed fully at $\sim 50 \%$ of the intersections ("closed gate intersections"). Gates closed partially, but without blocking the subject's route, at the remaining ( $\sim 50 \%)$ intersections ("open gate intersections"). The traffic signals correctly predicted the impending gate closure state at $\sim 80 \%$ of the gates, displaying red (Stop) for closed-gate intersections and green (Go) for opengate intersections. Traffic signals were incorrect (misleading) for the remaining ( $20 \%)$ gates, displaying green (Go) for closed-gate intersections and red (Stop) signals for open-gate intersections. Correct and misleading signals were randomly interspersed. So, while traffic signals were usually correct, the truthfulness of each specific signal was not known to the driver.

Each subject drove through a series of 103 intersections. The first three intersections were designated for warm-up and training. After becoming familiar with the experimental setup (including the task and the steering wheel, accelerator pedal and brake controls), the subject was instructed to travel through the next 100 intersections as quickly as possible without hitting the gates, using the traffic signals to help.

\section{INITIAL EXPERIMENTAL RESULTS}

Thirty-two licensed drivers participated in a study using the Go/No-Go scenario tool. Fourteen were recruited from the Iowa Patient Registry of patients with behavioral impairments due to chronic focal brain lesions, two had early Alzheimer's disease, and the rest were neurologically normal. All subjects were tested in detail on a battery of tests of perception, attention, memory, language, and decision-making in the Benton Neuropsychology Laboratory (Tranel, 1996; Rizzo et al., 1997b; 2001). 


\section{Hypotheses}

Neurologically impaired subjects would (1) take longer to complete the Go/No-Go scenario, (2) make a higher proportion of errors, and (3) improve less across trials (measured by comparing performance on intersections range $1-25$ vs $26-50$ vs $51-75$ vs $76-100$ ) than the neurologically normal controls.

\section{Results}

Tabulated results are contained in the tables at the end of the paper. Neurologically impaired subjects performed worse than the controls, took longer to go through the task, and had more collisions with gates, as hypothesized. Dependent measures included time-to-completion, number of crashes into closed gates, number of stops at open gates, and number of successes (i.e., stopping at closed gates and going at open gates).

Notably, subject 2458BH with a right frontal lesion (Figure 3), the well-documented subject EVR with bilateral frontal lobe amputations following resection of a meningioma (see e.g., Damasio et al., 1985; Eslinger and Damasio, 1985), and subject 1951RH, with herpes simplex encephalitis affecting mesial-temporal lobes bilaterally and right orbito-frontal cortex (Tranel et al., 2000), took the longest time to complete the task. 2458BH and EVR tended to stop at the open gates (Stop failures; "I don't trust them"), whereas 1951RH obeyed the signal slavishly, and stopped even when it was possible to go safely. In contrast, subject 2771DC, who had a right temporo-occipital lesion, and subject 2720LB, who had developmental frontal lobe lesions, had shorter times-to-completion but had the most crashes into closed gates (Go failures).

Neurologically normal drivers infrequently had failures when the gates were open (thus they had very high Go successes and very low Stop failures), though some did hit closed gates (Go failures). Neurologically normal subjects completed the test relatively quickly. Among the subjects with lesions, the two fastest had very high incidences of Go failures (crashing into closed gates).

Also of note, drivers with executive function impairment who had crashes at gates and took longer to get through the task continued to show good control of the vehicle and did not exceed the lane boundaries of the road. In other words visuomotor control can be intact in drivers with decision-making impairment, suggesting that measures of visuomotor control in the driving task 
(such as steering and lane position variability) alone are not sensitive predictors of critical incidents caused by decision-making impaired drivers.

Furthermore, drivers who had lesions in areas that did not cause executive dysfunction performed well on the Go/No-Go task, supporting the specificity of this task in localizing decision-making impairments in a driving-like task.

\section{DISCUSSION}

Our overall goal is to develop new tools for evaluating decision-making impairments in driving. Previously available testing resources ranged from standardized neuropsychological tests to high-end driving simulators. Driving simulation offers several advantages over the use of road tests or driving records in assessments of driver fitness. Simulator studies provide the only means to replicate exactly the experimental road conditions under which driver decisions are made, and simulations are safe, without the safety risks of the road or test track. But current driving simulators are relatively expensive to use and maintain and remain impractical to deploy widely for driver impairment testing.

On the other hand, standard neuropsychological tests can fail to reveal decision-making impairments that patients make in real-life activities such as automobile driving (Rizzo, 2003). In this context, we developed an abstract virtual environment tool that captures essential elements of driving-related decision-making, while being deployable on single-screen commodity PC systems.

Our approach draws from perceptual psychology, computer graphics, art and human factors to provide appropriate visual perceptual cues. We deviated from traditional approaches used in driving simulation, focusing on what was needed for our intended assessment rather than assuming that visual realism was necessary. The environment was designed to provide sufficient pictorial and motion cues relevant to the task of perceiving the spatial relationships of objects and the orientation of the user in the simulated environment (Wanger et al., 1992; Palmer, 1999; Cutting, 1997). Similar to high-fidelity driving simulators and avionics synthetic vision systems, it utilizes motion parallax, optical flow caused by moving objects and the observer, shading, texture gradients, relative size, perspective, occlusion, convergence of parallel lines, and position of objects relative to the horizon.

The results of the pilot tests are promising. Significant differences between impaired and control subjects, not available from standard neuropsychological tests, were demonstrated in the Go/NoGo scenario. The finding of a shallower learning curve across Go/No-Go trials in brain-damaged drivers suggests a failure of response selection criteria based on prior experience, as previously reported in brain-damaged individuals with decision-making impairments on a gambling-related task (e.g., Bechara et al., 1997).

We are further developing the software to provide flexibility of scenario details (route, number of intersections, distance between intersections, trigger points, etc.) and to contain components usable for other tests. In particular, we will next develop and conduct experiments using a "Mudsplash" scenario (O'Regan et al., 1999) to assess subjects' susceptibility to distractions. 
Size, luminance, location, and timing of the display of potential distractors will be varied with respect to Go/No-Go events.

\section{Tables}

\section{Hypothesis 1}

\begin{tabular}{|c|c|c|c|c|c|c|c|c|c|}
\hline & \multicolumn{4}{|c|}{$\begin{array}{l}\text { Time in seconds to complete scenario } \\
\text { Neurologically Impaired }(\mathrm{n}=16)\end{array}$} & \multicolumn{4}{|c|}{ Controls $(n=16)$} & \\
\hline & Mean(SD) & Min & Median & $\operatorname{Max}$ & Mean(SD) & Min & Median & Max & $P$ value \\
\hline Time quarter 1 & $384.5(58.6)$ & 318.7 & 375.0 & $\overline{518.0}$ & $342.0(23.7)$ & 312.7 & 340.5 & 397.7 & .012 \\
\hline Time quarter 2 & $425.4(127.2)$ & 283.2 & 392.1 & 817.6 & $366.8(55.7)$ & 334.0 & 349.0 & 562.5 & .003 \\
\hline Time quarter 3 & $408.9(131.8)$ & 332.8 & 368.5 & 864.3 & $350.0(44.7)$ & 319.0 & 341.8 & 513.9 & $<.001$ \\
\hline Time quarter 4 & $366.9(90.6)$ & 316.1 & 337.9 & 691.2 & $323.8(16.2)$ & 229.5 & 321.2 & 366.6 & .006 \\
\hline Time total & 1585(390.8) & 1282 & 1471 & 2875 & $1382(130.0)$ & 1290 & 1366 & 1841 & .002 \\
\hline
\end{tabular}

$\mathrm{P}$ values from Wilcoxon Rank Sum tests. Conclusion: Mean times to complete each quarter as well as the entire task were significantly higher in neurologically impaired subjects

\section{Hypothesis 2}

\begin{tabular}{|c|c|c|c|c|c|c|c|c|c|c|}
\hline \multirow[t]{3}{*}{ 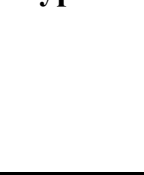 } & \multicolumn{10}{|c|}{ Number of failures and successes in impaired (NI) and control (C) subjects } \\
\hline & \multicolumn{2}{|c|}{ Go failure* } & \multicolumn{2}{|c|}{$\begin{array}{l}\text { Stop } \\
\text { failure* }\end{array}$} & \multicolumn{2}{|c|}{ Go Success* } & \multicolumn{2}{|c|}{ Stop Success* } & \multicolumn{2}{|c|}{ Other Success* } \\
\hline & NI & $\mathrm{C}$ & NI & $\mathrm{C}$ & NI & $\mathrm{C}$ & NI & $\mathrm{C}$ & $\mathrm{NI}$ & $\mathrm{C}$ \\
\hline quarter 1 & 37 & 36 & 36 & 9 & 187 & 215 & 138 & 134 & 2 & 8 \\
\hline quarter 2 & 45 & 37 & 32 & 8 & 128 & 152 & 185 & 190 & 10 & 15 \\
\hline quarter 3 & 22 & 14 & 26 & 0 & 150 & 176 & 179 & 189 & 22 & 21 \\
\hline quarter 4 & 22 & 17 & 24 & 2 & 200 & 222 & 133 & 129 & 21 & 30 \\
\hline Total & 126 & 100 & $\overline{111}$ & 19 & 665 & 765 & 635 & 642 & 55 & 74 \\
\hline
\end{tabular}

*Go failure $=$ go when should stop (closed gate) Stop failure=stop when should go (open gate)

Go success $=$ go when should go (open gate) Stop success=stop when should stop (closed gate) Percent go failures*

\begin{tabular}{l|llll|lllll|l} 
& \multicolumn{2}{|l|}{ Neurologically Impaired $(\mathrm{n}=16)$} & \multicolumn{3}{l|}{ Controls $(\mathrm{n}=16)$} & \\
\hline & Mean(SD) & Min & Median & Max & Mean(SD) & Min & Median & Max & P value \\
\hline \% go failure q1 & $.212(.134)$ & .092 & .182 & .545 & $.200(.111)$ & 0 & .182 & .454 & .786 \\
\hline \% go failure q2 & $.193(.198)$ & 0 & .177 & .786 & $.150(.105)$ & 0 & .133 & .333 & .805 \\
\hline \% go failure q3 & $.105(.091)$ & 0 & .110 & .357 & $.067(.093)$ & 0 & 0 & .286 & .217 \\
\hline \% go failure q4 & $.132(.145)$ & 0 & .095 & .545 & $.125(.111)$ & 0 & .091 & .333 & $1.00 ? ?$ \\
\hline$\%$ total go failure & $.162(.118)$ & .030 & .150 & .440 & $.133(.072)$ & 0 & .130 & .255 & .624 &
\end{tabular}

$* \%$ go failure $=$ go failure/(go failure+stop success)

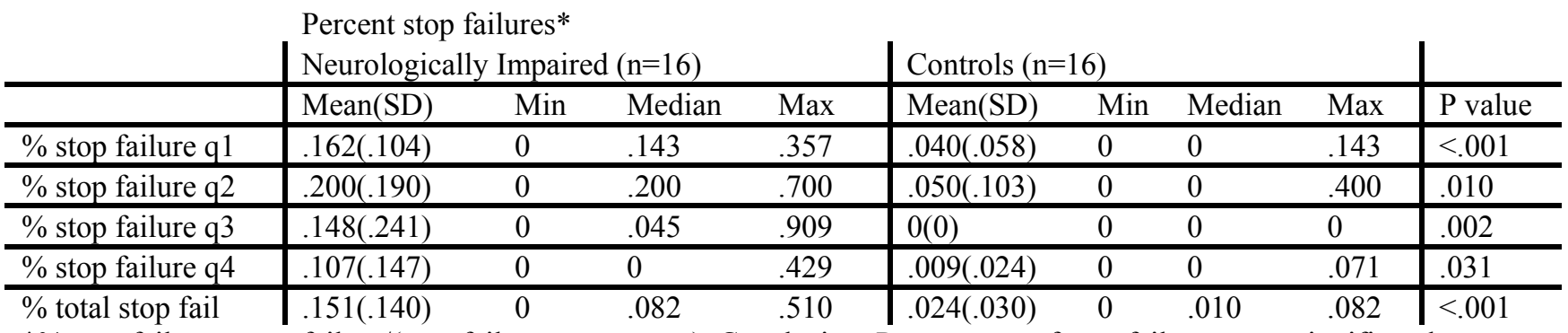

$* \%$ stop failures $=$ stop failure/(stop failure+go success). Conclusion: Percentages of stop failures were significantly

higher for the neurologically impaired subjects, but there were no differences between groups in go failures. 


\section{Hypothesis 3}

Failures across time

Slope: impaired

Slope: controls
Mean slope $(95 \% \mathrm{CI})$ $-.027(-.046,-.009)$

$-.025(-.039,-.011)$
Slope $=($ go failure + stop failure $) /($ go failure + stop failure+go success+stop success+other success). Conclusion: Least squares slope across time indicated improvement in both groups, but improvement between groups was not significantly different $(p=.720)$

\section{REFERENCES}

Bechara A., Damasio H., Tranel D., Damasio, A.R. (1997). Deciding advantageously before knowing the advantageous strategy. Science, 275, 1293-1295.

Brooks, F.P. (1999). What's real about virtual reality? IEEE Computer Graphics and Applications, 19, 16-27.

Brower, W.H., Ponds, R.W.H.M., Van Wolfelaar, P.C., Van Zomeran, A.H. (1989). Divided attention 5 to 10 years after severe closed head injury. Cortex, 25, 219-30.

Cutting, J.E. (1997). How the eye measures reality and virtual reality. Behavior Research Methods, Instrumentation, and Computers, 29, 29-36.

Damasio, A.R., Eslinger, P.J., Damasio, H., et al. (1985). Multimodal amnesic syndrome following bilateral temporal and basal forebrain damage. Arch Neurol, 42, 252-259.

Ellis, S.R. (2000). On the design of perspective displays. Proceedings: $15^{\text {th }}$ Triennial Conference, International Ergonomics Association/44th Annual Meeting, Human Factors \& Ergonomics Society, San Diego, CA, 2000. Santa Monica, CA: HFES.

Ellis, S.R. (1993). Pictorial communication: Pictures and the synthetic universe. Ch. 2. In: Ellis, S.R. (ed.) Pictorial Communication in Virtual and Real Environments. 2nd ed. London; Washington DC: Taylor \& Francis.

Eslinger, P.J., Damasio, A.R. (1985). Severe disturbance of higher cognition after bilateral frontal lobe ablation: patient EVR. Neurology, 35, 1731-1741.

Guerrier, J.H., Manivannan, P., Pacheco, A., et al. (1995). The relationship of age and cognitive characteristics of drivers to performance of driving tasks on an interactive driving simulator. Proceedings of the Human Factors and Ergonomics Society 39th Annual Meeting, San Diego. HFES, pp.172-176.

Haraldsson, P-O, Carenfelt, C., Laurell, H., Tornros, J. (1990). Driving vigilance simulator test. Acta Otolaryngol (Stockholm), 110, 136-40.

Madeley, P., Hully, J.L., Wildgust, H., Mindham, R.H.S. (1990). Parkinson's disease and driving ability. JNNP, 53, 580-582.

Palmer, S.E. (1999). Vision Science: Photons to Phenomenology. Cambridge, MA: MIT Press, 1999.

O'Regan, J.K., Rensink, R.A., Clark, J.J. (1999). Change-blindness as a result of 'mudsplashes.' Nature, 398, 34.

Rizzo M. Safe and unsafe driving. Ch. 9. In: Rizzo, M., Eslinger, P.J., eds. Principles and Practice of Behavioral Neurology and Neuropsychology. Philadelphia: WB Saunders, 2003.

Rizzo, M., Jermeland, J., Severson, J. (2002). Instrumented vehicles and driving simulators. In: Ball, K., Wahl, H-W (eds.) Driving in old age: Use of technology to promote independence. Gerontechnology, 1, 291-296. 
Rizzo, M., McGehee, D.V., Dawson, J., Anderson, S. (2001). Simulated car crashes at intersections in drivers with Alzheimer's disease. Alzheimer Dis Assoc Disord, 15, 10-20.

Rizzo, M., McGehee, D.V., Dingus, T.A., Petersen, A.D. (1997a). Development of an unobtrusively instrumented field research vehicle for objective assessments of driving performance. In: J. Rothengatter, E. Vaya Carbonell (eds.) Traffic and Transport Psychology: Theory and Application. Oxford: Pergamon, pp. 203-208.

Rizzo, M., Reinach, S., McGehee, D.V., Dawson, J. (1997b). Simulated car crashes and crash predictors in drivers with Alzheimer's disease. Arch Neurol, 54, 545-553.

Theunissen, E. (1997). Integrated Design of a Man-Machine Interface for 4-D navigation. Delft, The Netherlands: Delft UP.

Tranel, D. (1996). The Iowa-Benton school of neuropsychological assessment. In: Grant, I., Adams, K.M. (eds.). Neuropsychological Assessment of Neuropsychiatric Disorders. $2^{\text {nd }}$ ed. New York: Oxford UP, pp. 81-101.

Tranel, D., Damasio, H., Damasio, A.R. (2000). Amnesia caused by herpes simplex encephalitis, infarction in basal forebrain, and anoxia/ischemia. Vol. 2. Memory and its Disorders. In: Boller, F., Grafman, J. (eds.). Handbook of Neuropsychology. 2nd ed. Amsterdam: Elsevier, pp. 85-110.

Wanger, L.R., Ferwerda, J.A., Greenberg, D.P. (1992). Perceiving spatial relationships in computer-generated images. IEEE Computer Graphics and Applications, 12, 44-58. 